\title{
Real time observer-based stator fault diagnosis for IM
}

\author{
Khadidja El Merraoui, Abdellaziz Ferdjouni, M'hamed Bounekhla \\ Department of Electonic, Laboratoire des Systèmes Electriques et Télécommande (LABSET), \\ Saad Dahlab University, Algeria
}

\begin{tabular}{|c|c|}
\hline Article Info & ABSTRACT \\
\hline $\begin{array}{l}\text { Article history: } \\
\text { Received Jul 10, } 2018 \\
\text { Revised May 2, } 2019 \\
\text { Accepted Jun 27, } 2019\end{array}$ & $\begin{array}{l}\text { This paper proposes a delta connected IM model that takes the Stator } \\
\text { winding Inter-Turn Short Circuit (SITSC) fault into account. In order to } \\
\text { detect the fault and evaluate its severity, an observer based FDI method is } \\
\text { suggested. It allows the generation of residual using Extended Kalman filter } \\
\text { (EKF). To overcome the problem of the EKF initialization, the cyclic } \\
\text { optimization method is applied to determine its tuning parameters. }\end{array}$ \\
\hline $\begin{array}{l}\text { Keywords: } \\
\text { Extended kalman filter } \\
\text { FDI } \\
\text { Induction motor } \\
\text { Residual generator } \\
\text { Stator inter-turn short circuit }\end{array}$ & $\begin{array}{l}\text { The advantage of the proposed approach is the real-time quantification of } \\
\text { the fault severity and the quick fault detection. Using numerical simulation } \\
\text { under both the healthy and the faulty conditions, the proposed IM model and } \\
\text { EKF-based FDI approach are confirmed. Experimental results obtained by } \\
\text { a real-time implementation on test-bench validate the simulated results. }\end{array}$ \\
\hline \multicolumn{2}{|l|}{ Corresponding Author: } \\
\hline $\begin{array}{l}\text { Khadidja El Merraoui, } \\
\text { Departement of Electronic, } \\
\text { Saad Dahlab University, } \\
\text { Route Soumaa, Blida 1, Algeria. } \\
\text { Email: kelmerraoui@gmail.com }\end{array}$ & \\
\hline
\end{tabular}

\section{INTRODUCTION}

The high reliability and reduced costs made the three-phase induction motor (IM) the most widely used electrical machine in industry. During his work life, it suffers from multiple stresses leading to stator inter-turn faults, rotor faults or bearing faults. Stator faults represent around $40 \%$ of the total number of all type of faults [1]. The stator inter-turn short circuit (SITSC) can be induced by excessive raise of temperature, mainly caused by supply voltage imbalance or by increased current due to repeated starting of the IM or load variation. If not quicly detected, SITSC can lead to catastrophic IM behavior. Thus, it is mandatory to detect SITSC at an early stage to prevent damage to equipment or personnel [2].

IM Condition monitoring received a lot of effort during last decades. One important part was conducted by treating available signal from IM terminal, mainly stator currents and voltages. Motor current signature analysis (MCSA) processes stator currents by using spectral analysis in order to detect particular frequencies indicating the fault occurrence. Though, high spectral resolution and accurate values of the IM slip are required, and the constant speed is considered. This approach was upgraded by introducing wavelet analysis with the objective to overcome limitations of the MCSA method [3]. Other strategies, widely applied for fault detection, are based on negative-sequence current [4] or negative-sequence impedance [5]. The so-called Park's vector approach is commonly used because of its inherent simplicity [3]. These methods process the stator currents and can detect mismatch of current amplitudes which can be caused by either fault or supply-voltage imbalances. However, negative sequence impedance can be produced by the IM intrinsic asymmetry also. Another point of view is presented in [6]; the behavior of the stator currents and the inner IM temperature in the case of an open phase fault are analysed to propose a protection system.

Second widely aplied approach to IM condition monitoring is model-based diagnosis, which is expected to provide better performances in solving FDI issue. Since IM model presents nonlinearity and a high coupling between stator and rotor sides, it provides significant amount of information on the rotor 
side. In [7-9] dedicated IM models are developed to take the fault effects into account. Thus, on-line fault detection and isolation (FDI) is achieved by Observer-based diagnosis approach [10,11]. Adaptive observers are also proposed in the literature $[12,13]$. However the implementation of this type of observer requires cumbersome calculations, which constitutes the drawback of this method. In [14, 15], Extended Kalman Filter (EKF) is used to estimate the rotor resistance, in order to detect broken bars. For stator related faults, the state variables and some IM model parameters are estimated recursively using EKFs allowing only the detection of such faults by simulation in $[16,17]$.

In this work a dedicated IM model is elaborated in which fault factor takes SITSC into account. A real time extended Kalman filter based FDI is proposed in order to detect SITSC by estimating fault factor and computing the fault severity. On the other hand, to efficiently tune EKF, the cyclic method [18] is applied to compute the noise covariance matrices to optimize the EKF working. Undeniably, the convergence and the quality of the estimation of EKF are highly influenced by the covariance matrices of state and output noises.

All experiments are conducted on a test-bed built around a dSPACE 1104 kit and a rewound IM fed on delta connected. This IM customized design allows working out different scenarios for healthy and faulty situations. The first experimental results validate the proposed IM model. Then, residual generation is carried out and permits both the detection of the SITSC and the evaluation of the fault severity on the faulty phase. The proposed real-time quantification of the fault severity in order to detect the incipient SITSC, to make decision on stopping or not the IM is achieved.

This paper is organised as follows: in Section 2 an enhanced IM model, taking into account of SITSC thanks to fault factor, is developed. In Section 3, the fault detection approach based on EKF is presented. Then, the cyclic method, applied in order to tune EKF, and the residual generator are developed. In Section 4, numerical simulation and implementation on a dSPACE 1104 kit are illustrated, to achieve the experimental validations of the IM model and the EKF-based FDI approach. A conclusion finishes this work.

\section{IM MODEL WITH STATOR WINDING FAULT}

In this section, an IM model which takes explicitly the SITSC in phase $A$ into account is developed. This model is used to analyze the IM behavior in healthy and faulty conditions. In the literature models describing IM with faults have been proposed in multiplicative form [7, 8, 12] or in additive form [10]. The IM model is developed based on the conventional assumptions (no saturation of magnetic circuit, sinusoidal distribution of magnetomotive forces ( $\mathrm{mmf}$ ) and skin effect neglected) [19]. The SITSC in phase $A$, as is showen in Figure 1, is described by the fault factor $f_{a}$ defined by (1) and is introduced in the IM model. $f_{a}$ is the quantity of healthy turns among the total number of turns in the stator phase $A$.

$$
\begin{aligned}
& n_{a}=\frac{n_{c c a}}{n_{s}} \\
& f_{a}=1-n_{a}
\end{aligned}
$$

$n_{c c a}$ is the number of shorted turns in phase $A . n_{s}$ is total number of turns.

Ideally, $f_{a}<1$ stands for the faulty case and $f_{a}=1$ stands for the no faulty one.

The stator resistance matrix $R_{s}$, the stator inductance matrix $M_{S S}$ and the stator-rotor mutual inductance $M_{s r}$ are modified consequently by introducing the fault factor $f_{a}$. They are given in the appendix A. The rotor resistance matrix $R_{r}$, the rotor inductance matrix $M_{r r}$ and the rotor-stator mutual inductance $M_{s r}$ are given in the appendix A also.

The following set of equations describes the IM model.

$$
\begin{aligned}
& U_{s}=R_{s} I_{s}+\frac{d}{d t}\left(\Phi_{s}\right) ; U_{r}=R_{r} I_{r}+\frac{d}{d t}\left(\Phi_{r}\right)=0 \\
& \Phi_{s}=M_{s s} I_{s}+M_{s r} I_{r} ; \Phi_{r}=M_{r r} I_{r}+M_{r s} I_{s}
\end{aligned}
$$

$I_{s}\left(I_{r}\right)$ and, $\Phi_{s}\left(\Phi_{r}\right)$ stand for the stator (rotor) currents and fluxes vectors respectively. $U_{s}$ and $\mathrm{U}_{r}$ stand for the stator and rotor voltages vectors respectively. 


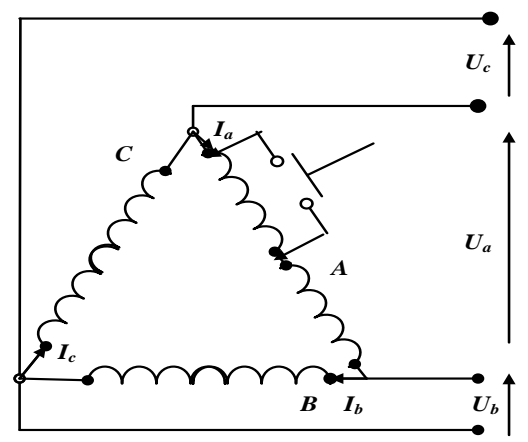

Figure 1. Representation of delta-connected IM with SITCS on phase $A$

By using (2) and (3), cited before, the state equation of IM model which takes the stator faults into account is then given by:

$$
\left(\begin{array}{c}
\dot{I}_{s} \\
\dot{\Phi}_{r}
\end{array}\right)=\left(\begin{array}{cc}
A_{1} & A_{2} \\
A_{3} & A_{4}
\end{array}\right)\left(\begin{array}{c}
I_{s} \\
\Phi_{r}
\end{array}\right)+\left(\begin{array}{c}
B_{0} \\
0_{3}
\end{array}\right) U_{s}
$$

Where:

$$
I_{s}=\left(\begin{array}{lll}
I_{s a} & I_{s b} & I_{s c}
\end{array}\right)^{T} ; \Phi_{r}=\left(\begin{array}{lll}
\Phi_{r a} & \Phi_{r b} & \Phi_{r c}
\end{array}\right)^{T} ; U_{s}=\left(\begin{array}{lll}
U_{a} & U_{b} & U_{c}
\end{array}\right)^{T} ;(.)^{T} \text { is the matrix transpose. }
$$

The matrices $A_{1}, A_{2}, A_{3}, A_{4}$ and $B_{0}$ are given thereafter:

$$
\begin{aligned}
& A_{1}=\left(M_{s s}-M_{s r} M_{r r}^{-1} M_{r s}\right)^{-1}\left(\dot{M}_{s r} M_{r r}^{-1} M_{r s}+M_{s r} M_{r r}^{-1} R_{r} M_{r r}^{-1} M_{r s}+M_{s r} M_{r r}^{-1} \dot{M}_{r s}-R_{s}\right), \\
& A_{2}=\left(M_{s s}-M_{s r} M_{r r}^{-1} M_{r s}\right)^{-1}\left(-\dot{M}_{s r} M_{r r}^{-1}-M_{s r} M_{r r}^{-1} R_{r} M_{r r}^{-1}\right), A_{3}=-R_{r} M_{r r}^{-1} M_{r s}, A_{4}=R_{r} M_{r r}^{-1}, \\
& B_{0}=\left(M_{s s}-M_{s r} M_{r r}^{-1} M_{r s}\right)^{-1}, 0_{3}: 3 \times 3 \text { zero matrix. }
\end{aligned}
$$

These matrices are not symmetric and depend on the angle $\theta$, which is time varying. To obtain a model with constant matrices, the $P$ transform described by (5) is applied to (4). This matrix is bloc-diagonal, invertible and orthogonal.

$$
P=\left(\begin{array}{ll}
P_{0} & 0_{3} \\
0_{3} & P_{1}
\end{array}\right)
$$

$P_{0}$ is the Concordia matrix and $P_{l}$ is the time-varying matrix defined below.

$$
P_{0}=\sqrt{\frac{1}{6}}\left(\begin{array}{ccc}
2 & -1 & -1 \\
0 & \sqrt{3} & -\sqrt{3} \\
\sqrt{2} & \sqrt{2} & \sqrt{2}
\end{array}\right) ; P_{1}=\sqrt{\frac{2}{3}}\left(\begin{array}{ccc}
c_{1} & c_{2} & c_{3} \\
t_{1} & t_{2} & t_{3} \\
\frac{1}{\sqrt{2}} & \frac{1}{\sqrt{2}} & \frac{1}{\sqrt{2}}
\end{array}\right)
$$

The coefficients $c_{1}, c_{2}, c_{3}, t_{1}, t_{2}$, and $t_{3}$ are given in the appendix A.

$$
P\left(\begin{array}{c}
\dot{I}_{s} \\
\dot{\Phi}_{r}
\end{array}\right)=\frac{d}{d t}\left(\begin{array}{c}
I_{s}^{*} \\
\Phi_{r}^{*}
\end{array}\right)=\left(\begin{array}{cc}
P_{0} A_{1} P_{0}^{-1} & P_{0} A_{2} P_{1}^{-1} \\
P_{1} A_{3} P_{0}^{-1} & \dot{P}_{1} P_{1}^{-1}+P_{1} A_{4} P_{1}^{-1}
\end{array}\right)\left(\begin{array}{c}
I_{s}^{*} \\
\Phi_{r}^{*}
\end{array}\right)+\left(\begin{array}{c}
P_{0} B_{0} P_{0}^{-1} \\
0_{3}
\end{array}\right) U_{s}
$$

Where:

$$
\begin{aligned}
& P\left(\begin{array}{l}
I_{s} \\
\Phi_{r}
\end{array}\right)=\left(\begin{array}{llllll}
I_{d s}^{*} & I_{q s}^{*} & I_{0 s}^{*} & \Phi_{d r}^{*} & \Phi_{q r}^{*} & \Phi_{0 r}^{*}
\end{array}\right)^{T}, I_{0 s}^{*}=0 ; \Phi_{0 r}^{*}=0 . \\
& \dot{X}(t)=\left(\begin{array}{cc}
P_{0} A_{1} P_{0}^{-1} & P_{0} A_{2} P_{1}^{-1} \\
P_{1} A_{3} P_{1}^{-1} & \dot{P}_{1} P_{1}^{-1}+P_{1} A_{4} P_{1}^{-1}
\end{array}\right) X(t)+\left(\begin{array}{c}
P_{0} B_{0} P_{0}^{-1} \\
0_{3}
\end{array}\right) U(t)
\end{aligned}
$$


After obvious calculation, the IM model which takes SITSC into account is obtained:

$$
\left\{\begin{array}{l}
\dot{x}_{1}(t)=\alpha_{0} x_{1}+\alpha_{1} x_{3}+\alpha_{2} \Omega x_{4}+\beta_{1} U_{d s} \\
\dot{x}_{2}(t)=\alpha_{3} x_{2}+\alpha_{4} \Omega x_{3}+\alpha_{5} x_{4}+\beta_{2} U_{q s} \\
\dot{x}_{3}(t)=\alpha_{6} x_{1}+\alpha_{7} x_{3}-\Omega x_{4} \\
\dot{x}_{4}(t)=\alpha_{8} x_{2}+\Omega x_{3}+\alpha_{7} x_{4}
\end{array}\right.
$$

Where the transformed state vector $X$ is defined as follow:

$$
X=\left(\begin{array}{llll}
x_{1} & x_{2} & x_{3} & x_{4}
\end{array}\right)^{T}=\left(\begin{array}{llll}
I_{d s}^{*} & I_{q s}^{*} & \Phi_{d r}^{*} & \Phi_{q r}^{*}
\end{array}\right)^{T}
$$

The parameters of (8) are defined by:

$$
\begin{gathered}
\alpha_{0}=q_{0}+\sqrt{2} q_{1} f_{a}+\sqrt{2} \frac{q_{2}}{f_{a}} ; \alpha_{1}=q_{3}\left(1+\frac{2}{f_{a}}\right) ; \alpha_{2}=\frac{q_{4}}{3}\left(1+\frac{2}{f_{a}}\right) ; \alpha_{3}=q_{3} ; \alpha_{4}=-q_{4} ; \alpha_{5}=3 q_{3} \\
\alpha_{6}=q_{5}+2 q_{5} f_{a}, \alpha_{7}=q_{6}, \alpha_{8}=3 q_{5}, \beta_{1}=q_{7}+2 \sqrt{2} q_{8} \frac{1}{f_{a}}+\sqrt{2} q_{9} \frac{1}{f_{a}^{2}}, \beta_{2}=q_{7}-q_{8} .
\end{gathered}
$$

The values of $q_{i}, i=1$ to 9 are given in the appendix A.

Electromagnetic torque, $C_{e}$ and Mechanical equation are given by the following expressions:

$$
\begin{aligned}
& C_{e}=\frac{M p}{3 M+2 l_{r}}\left(3 x_{2} x_{4}-\left(2 f_{a}+1\right) x_{1} x_{4}\right) \\
& J \frac{d}{d t}=C_{e}-f_{v} \Omega-C_{r}
\end{aligned}
$$

$J$ is the moment of inertia, $f_{v}$ is the viscous friction coefficient, and $p$ is the pole pairs number. $C_{e}$ and $\mathrm{C}_{r}$ are the electromagnetic and the load torque respectively.

- Remark 1:

In the case when no fault occurs the healthy IM model is retrieved and $f_{a}=1 \Leftrightarrow \alpha_{3}=\alpha_{0} ; \alpha_{4}=$ $\alpha_{2} ; \alpha_{5}=\alpha_{1} ; \beta_{2}=\beta_{1}$ and $\alpha_{8}=\alpha_{6}$

- Remark 2:

The IM model shown by (8) provides a simulation tool which permits analysis of several scenarios of healthy and faulty IM, avoiding the intrinsically destructive nature of inter-turn short-circuits. A stochastic IM model is computed taking into account of noise effects on state and output variables. The noises are assumed to be zero-mean, white and Gaussian also they are uncorrelated between them. Under the assumption that the rotor speed is measured and is constant between two sampling times, the discretization of the stochastic IM model is derived using second order Taylor expansion with a sample time $t_{s}$ [18]. The final IM model (9) is under a multiplicative fault form and is supposed to be detectable. It is used to design and implement the EKF for jointly estimate the fault factor on phase $A$ and the state vector.

$$
\left\{\begin{array}{c}
X_{k+1}=F\left(X_{k}, f_{a}, U_{k}\right)+W_{d_{k}} \\
Y_{k}=C X_{k}+V_{d_{k}}
\end{array}\right.
$$

Where: $X_{k}=\left(\begin{array}{lllll}x_{1_{k}} & x_{2_{k}} & x_{3_{k}} & x_{4_{k}}\end{array}\right)^{T}=\left(\begin{array}{lllll}I_{d s_{k}}^{*} & I_{q s_{k}}^{*} & \Phi_{d r_{k}}^{*} & \Phi_{q r_{k}}^{*}\end{array}\right)^{T}$ and $Y_{k}=\left(\begin{array}{ll}I_{d s_{k}}^{*} & I_{q s_{k}}^{*}\end{array}\right)^{T} \cdot W_{d_{k}}$ and $V_{d_{k}}$ is the discretized covariance of state and measurement noise matrices respectively. $W_{d_{k}} \in \mathbb{R}^{4} \times \mathbb{R}^{4}$ and $V_{d_{k}} \in \mathbb{R}^{2} \times \mathbb{R}$. The discretized input vector is: $U_{k}=\left(\begin{array}{ll}U_{d s k} & U_{q s k}\end{array}\right)^{T}$ and $=\left(\begin{array}{ll}I_{2} & 0_{2}\end{array}\right)$.

\section{EKF- BASED FDI APPROACH}

In this section, we are interested in the sequential processing of the EKF as a solution for the fault diagnosis. EKF is a stochastic nonlinear observer able of estimating the state vector and some model parameters. It is proposed in the FDI approach as it is adequate for a quick estimation. EKF is developed to estimate state variables and the fault factor $\boldsymbol{f}_{\boldsymbol{a}}$ by considering the extended state vector given in (11). EKF algorithm applied for the improved IM model (9) and extended to fault factor $\boldsymbol{f}_{\boldsymbol{a}}$, is given bellow [20, 21], with the assumption that $\boldsymbol{f}_{\boldsymbol{a}}$ has no dynamic (15). 


$$
\begin{aligned}
& X_{e_{k}}=\left(\begin{array}{lllllll}
x_{1_{k}} & x_{2_{k}} & x_{3_{k}} & x_{4_{k}} & 1
\end{array}\right)^{T}=\left(\begin{array}{lllllll}
I_{d s_{k}}^{*} & I_{q s_{k}}^{*} & \Phi_{d r_{k}}^{*} & \Phi_{q r_{k}}^{*} & 1
\end{array}\right)^{T} \\
& \hat{X}_{e_{k}}=\left(\begin{array}{lllllll}
\hat{x}_{1} & \hat{x}_{2} & \hat{x}_{3} & \hat{x}_{4} & \hat{f}_{a}
\end{array}\right)^{T}=\left(\begin{array}{llllll}
\hat{I}_{d s}^{*} & \hat{I}_{q s}^{*} & \widehat{\Phi}_{d r}^{*} & \widehat{\Phi}_{q r}^{*} & \hat{f}_{a}
\end{array}\right)^{T}
\end{aligned}
$$

a. Initialization step:

$$
\hat{X}_{e 0}=\left(\begin{array}{lllll}
\hat{I}_{d s_{0}}^{*} & \hat{I}_{q s_{0}}^{*} & \widehat{\Phi}_{d r_{0}}^{*} & \widehat{\Phi}_{q r_{0}}^{*} & 1
\end{array}\right)^{T} ; P_{k}=P_{0}
$$

b. Prediction step:

$$
\begin{aligned}
& \hat{X}_{e_{k+1 / k}}=F\left(\hat{X}_{e_{k}}, \Omega, \hat{f}_{a}, U_{k}\right) \\
& P_{k+1 / k}=\left(\left.\frac{\partial F_{e}}{\partial \hat{X}_{e}}\right|_{\hat{X}_{e_{k+1} / k}}\right) P_{k}\left(\left.\frac{\partial F_{e}}{\partial \hat{X}_{e}}\right|_{\hat{X}_{e_{k+1} / k}}\right)^{T}+W_{d_{k}} \\
& \text { Where: } F_{e}\left(\hat{X}_{e_{k}}, \Omega ; \hat{f}_{a}, U_{k}\right)=\left(\begin{array}{cc}
F\left(\hat{X}_{e_{k}}, \Omega, U_{k}\right. & 0_{4 \times 1} \\
0_{1 \times 4} & 1
\end{array}\right) X_{e_{k}}
\end{aligned}
$$

and $P_{k} \in \mathbb{R}^{5} \times \mathbb{R}^{5}$ and $(.)^{T}$ is the matrix transpose.

c. Computations of EKF gain step and correction step:

$$
\begin{aligned}
& K_{k / k}=P_{k+1 / k} C_{d}^{T}\left(C_{d} P_{k+1 / k} C_{d}^{T}+V_{d_{k}}\right)^{-1} \\
& \hat{X}_{e_{k / k}}=\hat{X}_{e_{k+1 / k}}+K_{k / k}\left(Y_{k / k}-\hat{Y}_{k+1 / k}\right) ; \hat{Y}_{k+1 / k}=C_{d} \hat{X}_{e_{k+1 / k}}
\end{aligned}
$$

d. Computation of the estimation error variance step:

$$
P_{k}=\left(I_{5}-K C_{d}\right) P_{k+1 / k}
$$

Where: $P_{k}=E\left[\left(X_{e_{k}}-\hat{X}_{e_{k}}\right)\left(X_{e_{k}}-\hat{X}_{e_{k}}\right)^{T}\right.$ is the mathematical expectation of the covariance of the estimation error matrix. $P_{k+1 / k}$ is the covariance of the prediction error matrix. $K_{k / k}$ is the EKF gain. $I_{5}$ is $(5 \times 5)$ identity matrix.

\section{Application of cyclic method for tuning EKF}

The accurate knowledge of the state and measurement noise covariance matrices is essential for the good functioning of EKF algorithm. The Trial-and-error method for EKF tuning is commonly used but can be tedious. To overcome this situation, heuristic approaches, such as a Memetic algorithm [22], PSO algorithm [20] or Genetic algorithm [23] were used to automatically optimize both the state and measurement noise matrices. In this work, the cyclic method [18], which is a simple and an efficient technique, is applied to determine the optimal state and measurement noises. It was used in [18] to solve the optimization problem encountered in the parameters Identification of the Switched-reluctance machine.

The covariance of the state noise matrix $W_{d}$ is assumed to be a constant diagonal matrix and the measurement noise $V_{d}$ a constant vector.

$$
W_{d}=\operatorname{diag}\left(w_{d i}, w_{d i}, w_{d \varphi}, w_{d \varphi}, w_{d f a}\right) ; V_{d}=\left(v_{d}, v_{d}\right)^{T}
$$

The cyclic technique use each coordinate of the vector $Z(19)$, successively, as the search direction in order to minimize the cost function $J(20)$ as described in the algorithm of the Figure 2.

$$
Z=\left(\begin{array}{llll}
w_{d i} & w_{d \varphi} & w_{d f a} & v_{d}
\end{array}\right)
$$

Where $w_{d i}, w_{d \varphi}$ and $w_{d f a}$ are the state noise auto-covariance of the phase stator currents, fluxes and fault factor respectively. $v_{d}$ is the measurement auto-covariance.

The EKF performance is evaluated by the cost function $J$ given in (20). 


$$
J=\min \frac{1}{N} \sum_{k=1}^{N}\left(I_{s d_{k}}-\hat{I}_{s d_{k}}\right)^{2}+\left(I_{s q_{k}}-\hat{I}_{s q_{k}}\right)^{2}
$$

$N$ is the number of samples by one iteration. The measured $\left(I_{s d_{k}}, I_{s q_{k}}\right)$ and estimated $\left(\hat{I}_{s d_{k}}, \hat{I}_{s q_{k}}\right)$ stator currents are introduced in the cost function $J$. Firstly, the EKF tuning method by the cyclic algorithm is applied in the case of healthy IM, as presented in the Figure 3. Numerous iterations are necessary to the cyclic algorithm to reach the optimal solution; thus, it must be run off line using the data measured on the test-bench. Then, the computed values of $W_{d}$ and $V_{d}$ are introduced in the EKF to estimate, on line, the extended state vector in the case of healthy and faulty IM. The studies of the obtained results with several applied SITSC, summarized in Table 1, show the effectiveness of the EKF tuned by the cyclic method.

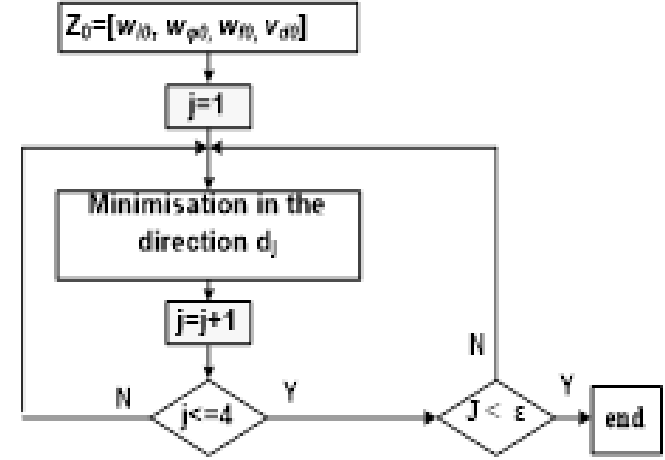

Figure 2. The cyclic method algorithm

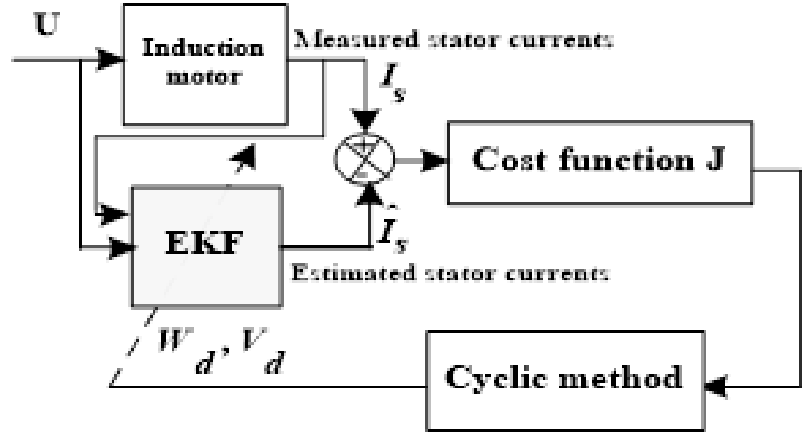

Figure 3. Representation of EKF tuning by a cyclic method

\section{RESIDUAL GENERATION}

FDI based on analytical redundancy consist of residual generation and decision making [24-26]. Residuals are quantities that represent the inconsistency between measured data and those delivered by the mathematical model or observer. Ideally, in normally case (no-fault) residual is equal to zero. However, state and measurement noises affect residuals and become different from zero. When a particular fault occurs, residual dedicated to this fault deviates from zero but not changes to other faults [26]. In this work, the residual consists of the deviation between the fault factor computed by EKF and its value in no-fault case, which is, ideally equal to (21).

$$
r=f_{a}-\hat{f}_{a}=1-\hat{f}_{a}
$$

By using (1) and (21), the residual $r$ is proportional to the ratio of shorted turns. Therefore, the fault severity, caracterised by the residual amplitude, is evaluated by means of these equations.

\section{EXPERIMENTAL RESULTS}

The proposed EKF-based FD is validated through simulation and experimental tests. In first stage, the improved IM mathematical model is validated; several scenario of the occurrence of the fault are experimented. The test-bench, given in Figure 4, consists of a three-phase squirrel cage IM, $0.55 \mathrm{~kW}, 380 \mathrm{~V}$, 4 poles. Each stator phase contains 4 coils of 132 turns connected in series. The IM is rewound, and a small amount of stator turn outputs are made to create a number of short circuits as shown in the Figure 5.

The IM stator windings are delta-connected and fed by a $50 \mathrm{~Hz}$ sinusoidal source voltage via a three-phase autotransformer. The rewound IM parameters are listed in the appendix B. The stator phase currents and voltages are measured via Hall-Effect sensors, and an encoder of 1024 counts/ $\mathrm{r}$ is used to measure the IM speed. Two A/D converters, 12 bits four channels and 16 bits four multiplexed channels, were used. The measured signals are filtered by anti-aliasing low pass filters, Buterworth type with $100 \mathrm{~Hz}$ cut frequency. EKF observer is implemented on the dSPACE1104 platform operating on a host PC linked to external signals by an interface board as shown in Figure 5. $C$-language is used to develop the EKF routines; which are compiled and uploaded into the host PC, with the functional sampling time $t_{s}=0.0004 \mathrm{~s}$. 


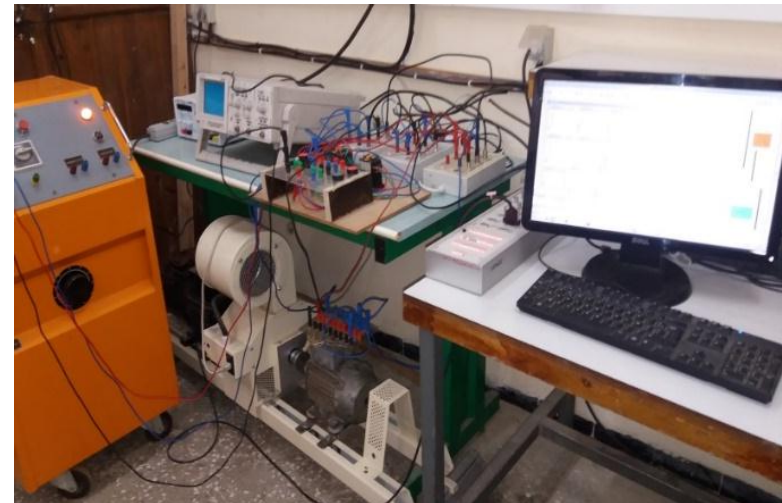

Figure 4. The test-bed

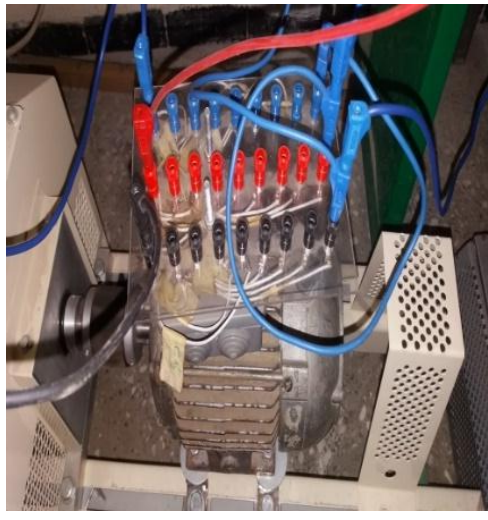

Figure 5. Rewound IM

The root mean square (RMS) values of the measured currents and those computed from IM model in both healthy and faulty cases are shown in Table 1. A well matching between them is shown and the difference is very small. These results permit the validation of the proposed IM model. Consequently, thereafter, the improved IM model can be used to perform the FDI based on EKF approach.

Table 1. RMS values of the measured and simulated currents on phase $A$

\begin{tabular}{cccccc}
\hline $\mathrm{f}_{\mathrm{a}}$ & $1-\mathrm{f}_{\mathrm{a}}$ & SITSC turns on phase $A$ & $\begin{array}{c}\text { SITSC } \\
\%\end{array}$ & $\overline{\mathrm{I}}_{\text {sa_m }}$ & $\bar{I}_{\text {Sa_s }}$ \\
\hline 1 & 0 & 0 & 0 & 1.05 & 1.07 \\
0.98 & 0.02 & 6 & $1.13 \%$ & 1.23 & 1.15 \\
0.95 & 0.05 & 24 & $4.54 \%$ & 1.5 & 1.42 \\
0.94 & 0.06 & 30 & $5.68 \%$ & 1.6 & 1.51 \\
\hline
\end{tabular}

Where: $\overline{\boldsymbol{I}}_{\boldsymbol{s} \boldsymbol{a} \_\boldsymbol{m}}$ is the RMS value of the measured stator current on phase $A$ of the rewound IM. $\overline{\boldsymbol{I}}_{\boldsymbol{s} \boldsymbol{a} \_\boldsymbol{s}}$ is the RMS value of stator current, on phase $A$, obtained by the proposed mathematical IM model.

\subsection{Experimental validation of EKF-based residual generation tuning by the Cyclic method}

In this section, the EKF-based FDI approach is experimentaly tested in the test-bed shown in Figure 4. EKF estimates state variables and fault factor and both residual generation and fault severity are calculated. The negative sequence of stator currents [4] is computed in order to confirm the unbalance.

$$
\left(\begin{array}{l}
I_{p} \\
I_{n} \\
I_{0}
\end{array}\right)=\left(\begin{array}{ccc}
1 & a & a^{2} \\
1 & a^{2} & a \\
1 & 1 & 1
\end{array}\right)\left(\begin{array}{l}
I_{s a} \\
I_{s b} \\
I_{s c}
\end{array}\right)
$$

$I_{p}$ : positive sequence stator current, $I_{n}$ : negative sequence stator current. $a=e^{j 2 \pi / 3}$

In the first stage, a unique fault, at different level of severity, occurs in the phase $A$ of the rewound IM. A quantity of 0 (healthy IM), 6, 24, and 30 turns are deliberately shorted on the rewound IM. The estimated fault factor $\hat{f}_{a}$ and the residual $r$, achieved with this scenario are shown in the Figure 6 (a) to Figure $8(\mathrm{a})$. In healthy case the residual is close to 0.0025 but is different from zero, du to the modelisation error and experimental disturbances. It grows to 0.0125 for 6 short-circuited turns $(1.13 \%)$. In the case of 24 short-circuited turns (4.54\%), it raises to 0.0375 and to 0.045 for 30 turns $(5.68 \%)$. These residual values are selected in steady state of residual $r$ as shown in Figure 6(b) to Figure 8(b). Ideally, the provoked SITSC provides the values of $\left(1-f_{a}\right)$ shown in Table 1 . Compared to the experimental results, a little and constant offset appairs. It is du to non modeled and measurement errors.

In Figure 6(c), Figure 7(c) and Figure 8(c), the measured and filtered stator currents are given. Each one shows the increase of the stator current in the phase $A$, once the SITSC was provoked. In Figure 6(d), Figure 7(d) and Figure 8(d), the negative sequence current, that illustrate the transient and steady state, are also given in the same situation. In healthy IM the negative sequence current is about $54 \mathrm{~mA}$. Once the SITSC of 6 turns is provoked it increases to $100 \mathrm{~mA}$; for 24 SITSC it grows to $153 \mathrm{~mA}$ and to 
$183 \mathrm{~mA}$ in the case of 30 SITSC. These results confirm clearly the SITSC occurrence by means of the rise of the negative sequence current.

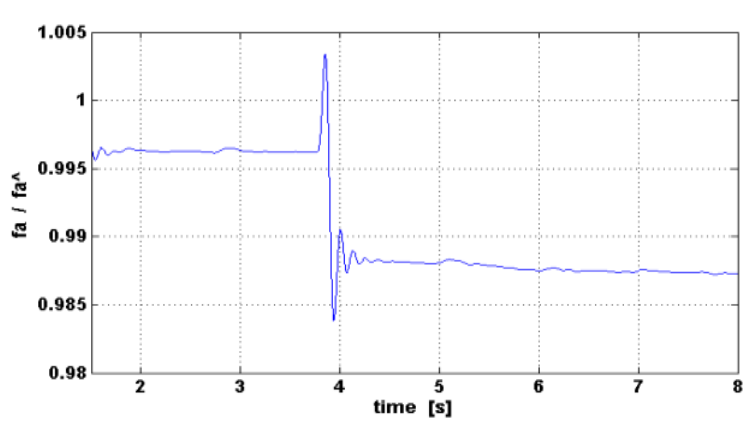

(a)

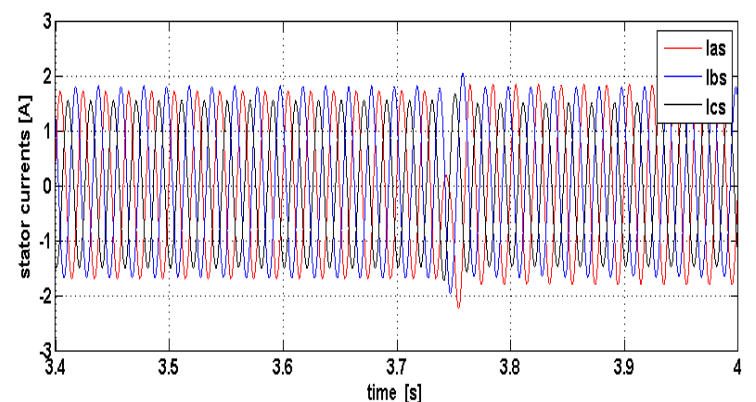

(c)

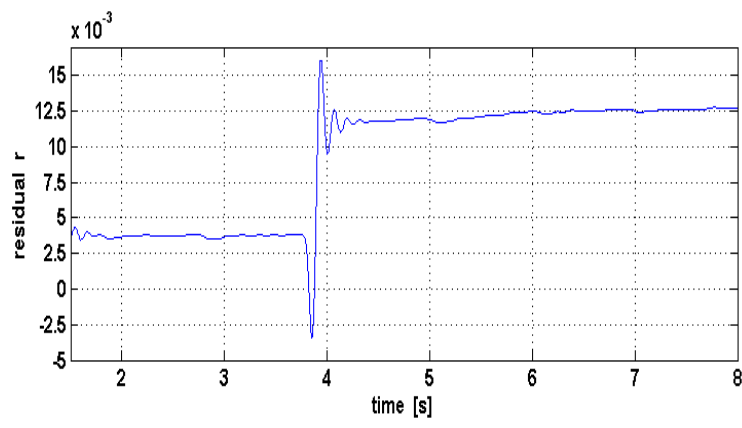

(b)

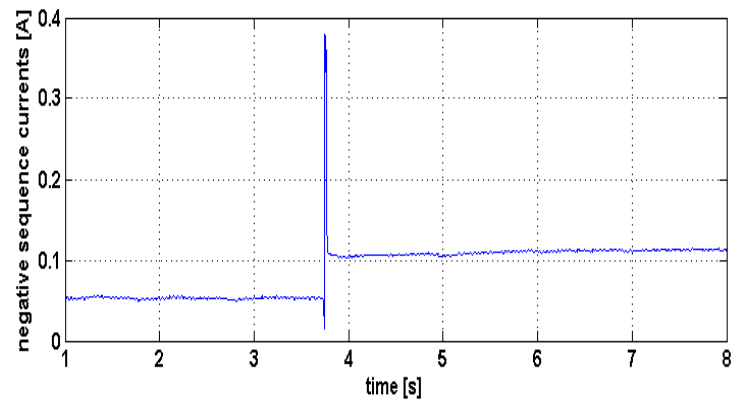

(d)

Figure 6. IM in Healthy case and SITSC of 6 turns, (a) $\hat{f}_{a}$ in healthy IM case and in SITSC of 6 turns, (b) Residual, (c) Filtred stator currents, (d) Negative sequence currents

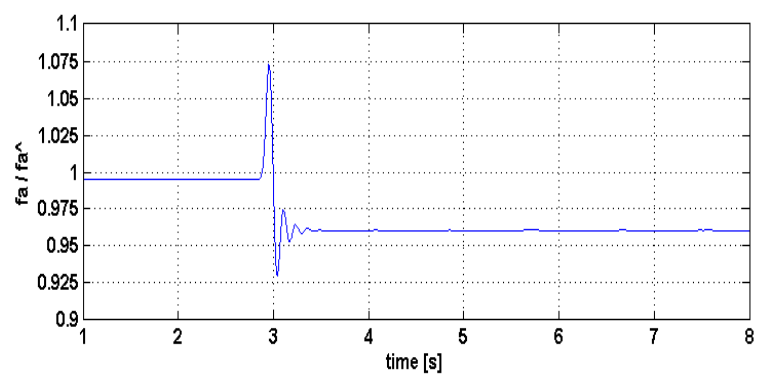

(a)

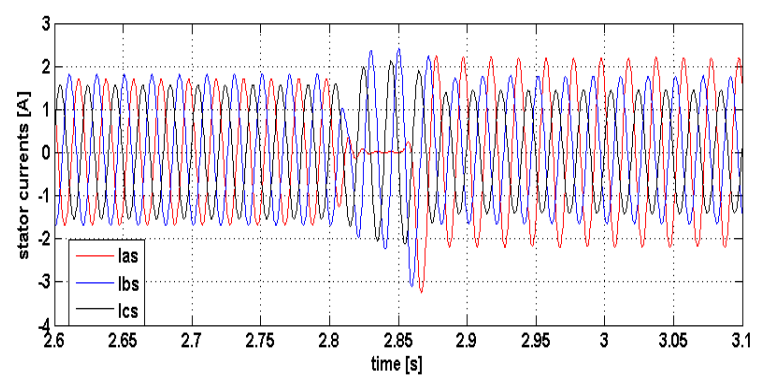

(c)

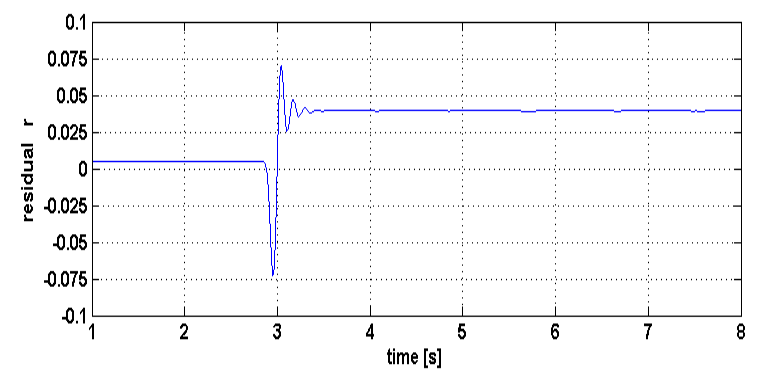

(b)

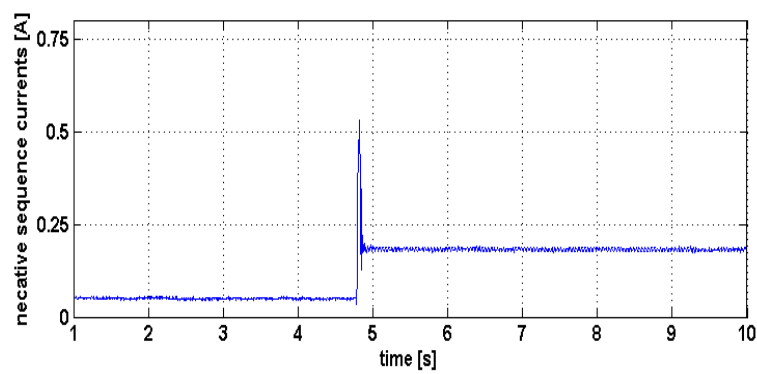

(d)

Figure 7. IM in healthy case and in SITSC of 24 turns, (a) $\hat{f}_{a}$ in healthy IM case and in SITSC of 6 turns, (b) Residual (c) Filtred stator currents, (d) Negative sequence currents 


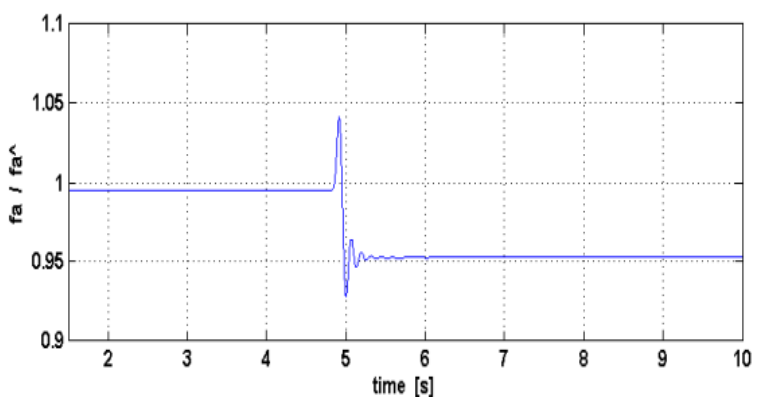

(a)

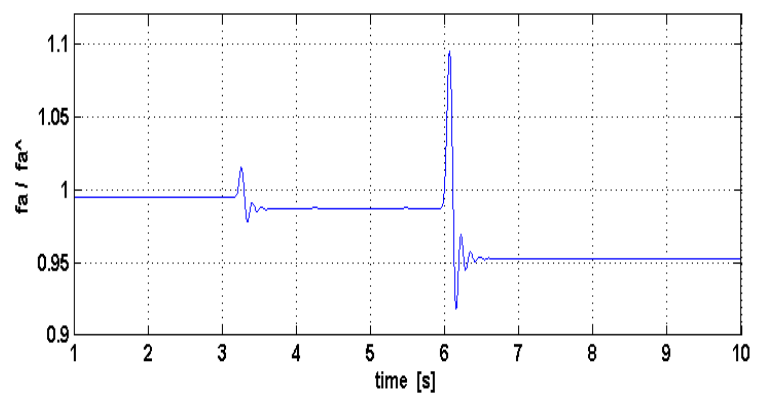

(c)

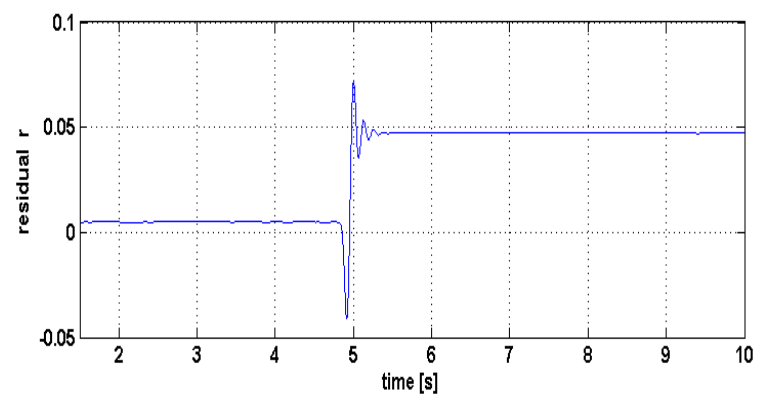

(b)

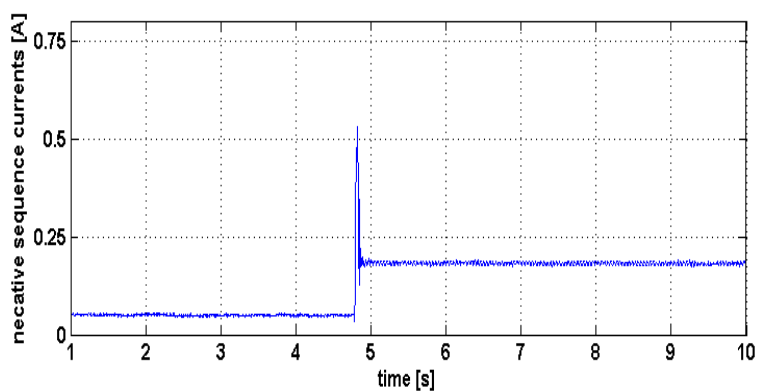

(d)

Figure 8. IM in healthy case and in SITSC of 30 turns, (a) $\hat{f}_{a}$ in healthy IM case and in SITSC of 30 turns, (b) Residual, (c) Filtred stator currents, (d) Negative sequence currents

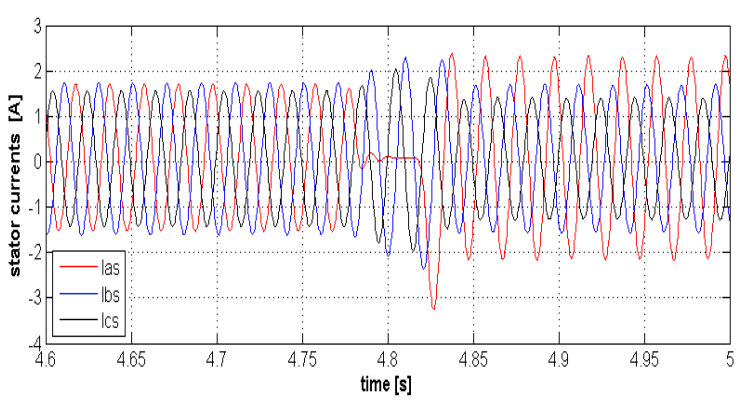

(a)

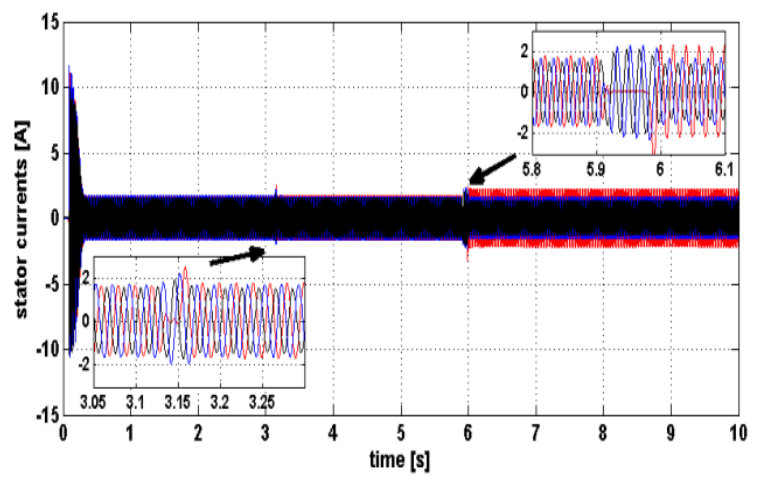

(c)

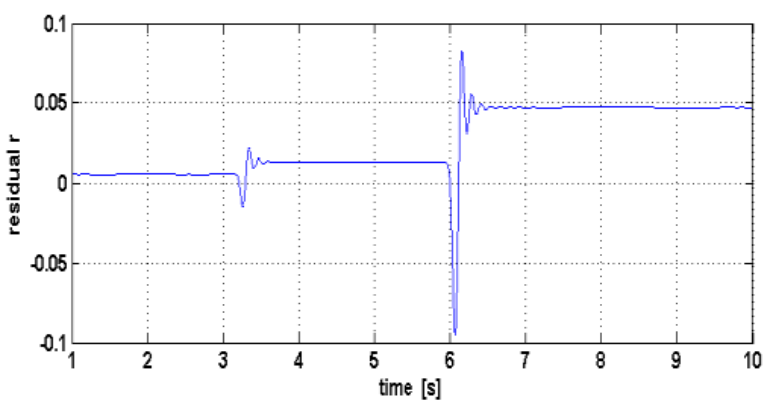

(b)

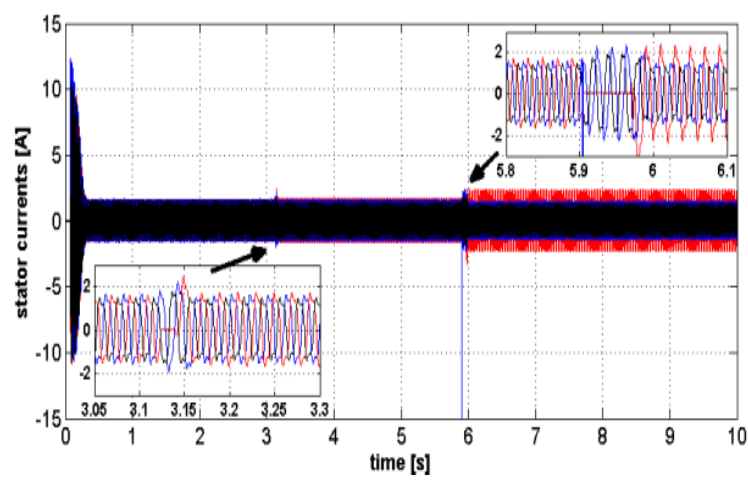

(d)

Figure 9. (a) $\hat{f}_{a}$ in healthy IM case and in SITSC of 6 and 30 turns, (b) Residual $r$, (c) No filtred stator currents in healthy IM and in 6 and 30 SITSC, (d) Filtred stator currents in healthy IM and in 6 and 30 SITSC 
The scenario implemented for the second set of fault is as follows: the IM is run healthy (without SITSC), after 3.12 seconds a fault of 6 SITSC is provoked, followed by a fault of 30 SITSC at about 4.9 seconds. Figure 9(a) to Figure 9(b) illustrate the obtained results by the test-bench. The fault factor $\hat{f}_{a}$ is well estimated by EKF; moreover, the residual $r$ indicates clearly the occurrence of the faults (Fault detection). The growth of the fault is well tracked by the residual $r$, which estimates its severity.

The three-phase stator currents recorded during this experience and the filtered ones are given by the Figure 9(c) and Figure 9(d). The repercussion of the provoked 6 SITSC on the rewound IM appears by a little rise on the magnitude of the faulty phase current. On the other hand, the current magnitude of the phase under a 30 SITSC increases more. Despite of the little jump of the stator current due to 6 SITSC, the evolution of the fault severity shows clearly the difference between this situation and the heathy IM. This fact is more significant in the residual $r$, which is more interesting in the sense that, it indicates the occurrence and the severity of the fault.

The residual $r$ obtained, in healthy situation, is close to zero, this is due to the good tuning of EKF by the cyclic method. The measured stator currents in the same fault condition and before filtering are given by Figure 9(c). They illustrate, in addition of the rise of the phase damaged current the deformation due to the noise harmonic. In all cases of SITSC considered above, the results show the good estimation of the fault factor and the residuals in comparison of the simulation values cited in the Table 1; this method permits the adequate evaluation of the fault severity and the early detection of the SITSC.

\subsection{Comparaison and discussion}

In these section, results of [10] are reviewed in order to highlight the advantage of the FDI based EKF approach presented in this work. In [10] a model of the induction motor, affine with respect to the SITSC has been developed. A sliding mode observer is designed, based on this IM model in the no fault case. Residuals generator is constructed on the basis of the error between the measured signals (the three phase currents) and variables provided by by the sliding mode observer.

Where: $\mathrm{x}_{\mathrm{i}}=\left(\begin{array}{lll}I_{s a} & I_{s b} & I_{s c}\end{array}\right)^{T}, \mathrm{z}_{\mathrm{i}}=\left(\begin{array}{lll}\hat{I}_{s a} & \hat{I}_{s b} & \hat{I}_{s c}\end{array}\right)^{T}$ and $r_{i}$ illustrates the residual equivalent to the phase $i$ $(i=\mathrm{a}, \mathrm{b}$ or $\mathrm{c}) . \mathrm{ni}$, is the ration of SITSC in phase $i$ forced at time $\mathrm{t}_{\mathrm{i}}$.

This method allows the detection and isolation of the fault as shown in Figure 10 and Figure 11. However, the quantification of the SITSC is unavailable. All the results presented in [10] are not experimentaly validated.
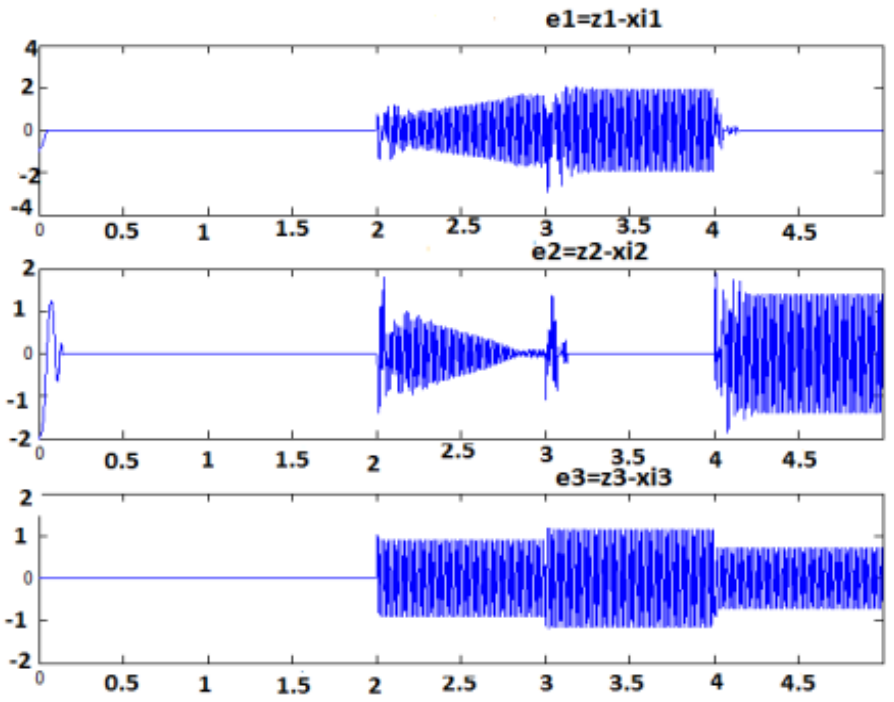

Figure 10. Errors between model and observer variables [10], (case with faults, na $=0.1, \mathrm{t}_{\mathrm{a}}=2 \mathrm{~s}, \mathrm{nb}=0.15, \mathrm{t}_{\mathrm{b}}=3 \mathrm{~s}, \mathrm{nc}=0.2, \mathrm{t}_{\mathrm{c}}=4 \mathrm{~s}$ ) 


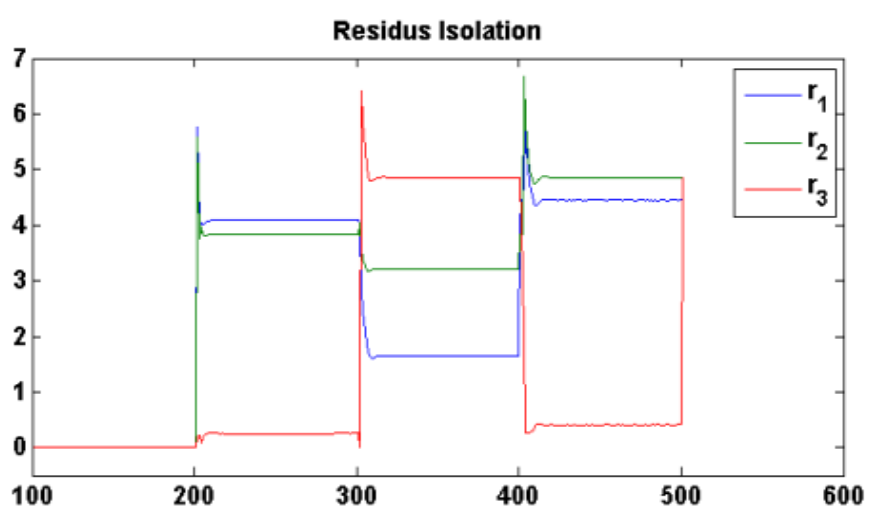

Figure 11. Faults isolation residuals $[10] \mathrm{r}_{1}, \mathrm{r}_{2}, \mathrm{r}_{3}$

\section{CONCLUSION}

Fault detection and diagnosis for induction motors is a wide area of research. The early SITCS detection in the IM is imperative for the protection of personnel and material. In this paper, FDI method based on EKF is proposed as a solution for the problem of early detection of SITSC. In a first step, an improved IM model, which takes into account of SITSC, is developed. The amount of SITSC is pointed out by the fault factor introduced in this model. An IM with customized design is built allowing the deliberate provokation of SITSC. After several experimentations, a good concordance between simulative and experimental results is achieved. Therefore, the proposed IM model is able to characterize the behavior of both healthy and faulty IM. The second step consists in the tuning of EKF by the cyclic method. EKF suffers from the lack of information about the noises. Then, cyclic method is carried out yielding accurate values of noise caracteristics, pemitting optimal working of EKF. The second contribution of this work is that the proposed EKF-based FDI method has been implemented (programmed in C-language) on dSPACE 1104 environment. The residual generation is obtained and the severity of the fault is evaluated on real time via the test-bench proving a high accuracy. The experimental results show the efficiency of the proposed EKFbased FDI method. As a perspective of this work, is the exploration of other faults like rotor broken bar and static or dynamic excentricity. Heuristic methods combined with the proposed EKF-based FDI approach seem to give promising results.

\section{APPENDIX A}

The matrices evolved in the proposed IM model described by (8) are detailed beneath,

$$
\begin{aligned}
& d_{0}=3 M+2 l_{r} ; d_{1}=3 M l_{r}+l_{s} d_{0} \\
& q_{0}=\frac{r_{s}\left(2 l_{r}^{2}\left(3 M-4 l_{s}\right)-d_{0} d_{1}-18 M^{2} l_{s}+3 M l_{r}\left(3 M-8 l_{s}\right)\right)-45 M^{2} r_{r} l_{s}}{9 d_{0} d_{1} l_{s}} ; q_{1}=-\frac{\sqrt{2} M^{2} r_{r}}{9 d_{0} d_{1}} \\
& q_{2}=\sqrt{2} \frac{r_{s} d_{0}\left(3 M l_{r}-d_{1}\right)-l_{S}\left(9 M^{2}\left(r_{r}+2 r_{s}\right)+8 r_{s} l_{r}\left(l_{r}+3 M\right)\right)}{9 d_{0} d_{1} l_{s}} ; q_{3}=-\frac{2 M r_{r}}{d_{0} d_{1}} ; q_{4}=\frac{M}{d_{1}} ; q_{5}=\frac{M r_{r}}{d_{0}} ; q_{6}=-\frac{2 r_{r}}{d_{0}} \\
& q_{7}=\frac{2 M l_{r}+d_{0} l_{s}}{3 l_{s}\left(d_{0} l_{s}+3 M l_{r}\right)} ; q_{8}=-\frac{\sqrt{2} M l_{r}}{l_{s}\left(3 l_{r}\left(2 l_{s}+3 M\right)+9 M l_{s}\right)} ; q_{9}=\frac{\sqrt{2}\left(M l_{r}+d_{0} l_{s}\right)}{3 l_{s}\left(d_{0} l_{s}+3 M l_{r}\right)} \\
& R_{S}=r_{s}\left(\begin{array}{ccc}
f_{a} & 0 & 0 \\
0 & 1 & 0 \\
0 & 0 & 1
\end{array}\right) ; M_{s r}=M\left(\begin{array}{ccc}
f_{a} c_{1} & f_{a} c_{2} & f_{a} c_{3} \\
c_{3} & c_{1} & c_{2} \\
c_{2} & c_{3} & c_{1}
\end{array}\right) ; M_{r s}=M_{s r}^{T} ; M_{s s}=M_{s}+L_{s \sigma} \\
& L_{s \sigma}=l_{s \sigma}\left(\begin{array}{ccc}
f_{a}^{2} & 0 & 0 \\
0 & 1 & 0 \\
0 & 0 & 1
\end{array}\right) ; M_{s}=M\left(\begin{array}{ccc}
f_{a}^{2} & -0.5 f_{a} & -0.5 f_{a} \\
-0.5 f_{a} & 1 & -0.5 f_{b} \\
-0.5 f_{a} & -0.5 f_{b} & 1
\end{array}\right), R_{r}=r_{r}\left(\begin{array}{ccc}
1 & 0 & 0 \\
0 & 1 & 0 \\
0 & 0 & 1
\end{array}\right) \\
& L_{r \sigma}=l_{r \sigma}\left(\begin{array}{ccc}
1 & 0 & 0 \\
0 & 1 & 0 \\
0 & 0 & 1
\end{array}\right) ; M_{r}=M\left(\begin{array}{ccc}
1 & -0.5 & -0.5 \\
-0.5 & 1 & -0.5 \\
-0.5 & -0.5 & 1
\end{array}\right) ; M_{r r}=M_{r}+L_{r \sigma} ; \\
& c_{1}=\cos (\theta)+0.5 ; c_{2}=\cos \left(\theta+\frac{2 \pi}{3}\right)+0.5 ; c_{3}=\cos \left(\theta-\frac{2 \pi}{3}\right)+0.5 \text { and } \theta: \text { Stator/rotor angle. }
\end{aligned}
$$

Where: $r_{s}, M$ and $l_{s \sigma}$ are the nominal values of the stator resistance, the mutual inductance and the stator leakage inductance, respectively. $r_{r}$ is the rotor resistance and $l_{r \sigma}$ is the rotor leakage inductance. 


\section{APPENDIX B}

The parameters of the IM used in Benchmark are given below. Stator resistance $r=13.63 \Omega$. Rotor resistance $r_{r}=13.31 \Omega$. Stator inductance $M_{s}=0.678 \mathrm{H}$, Rotor inductance $M_{r}=0.678 \mathrm{H}$. Stator leakage inductance $l_{s \sigma}=0.039 \mathrm{H}$, Rotor leakage inductance $l_{r \sigma}=0.039 \mathrm{H}$. Maximal value of the mutual inductance $M=0.664 \mathrm{H}$. Viscous friction coefficient $f_{v}=0.000643 \mathrm{Ns} / \mathrm{rad}$, Moment of inertia $J=0.002 \mathrm{~kg} \mathrm{~m}^{2}$. Rated speed $\Omega=1440 \mathrm{rpm}$. Rated phase current $I=1.6 \mathrm{~A}$. Rated line voltage $U=380 \mathrm{~V}$. Rated Power $P=0.55 \mathrm{~kW}$. Pole pairs number $p=2$.

\section{REFERENCES}

[1] IAS MR Working Group, "Report of large motor reliability survey of industrial and commercial installation," IEEE Transaction Industry Applications, vol. I-A-21, pp. 853-864, 1985.

[2] S. Nandi, "Detection of stator faults in induction machines using residual saturation harmonics," IEEE Transaction on Industry Applications, vol. 42, no. 5, pp. 1201-1208, 2006.

[3] S. K. Ahmed, et al., "DSP implementation of a novel envelope analysis approach for the diagnosis of broken bar in induction motor," Int. J. Modelling, Identification and Control (IJMIC), vol. 22, no. 3, pp. 275-284, 2014.

[4] Q. Wu and S. Nandi, "Fast single-turn sensitive stator inter-turn fault detection of induction machines based on positive-and negative-sequence third harmonic components of line currents," IEEE Transactions on Industry Applications, vol. 46, no. 3, pp. 974-983, 2010.

[5] J. L. Kohler, et al., "Condition monitoring of stator windings in induction motors: part i-experimental investigation of the effective negative-sequence impedance detector," IEEE Transactions on Industry Applications, vol. 38, no. 5, pp. 1447-1453, 2002.

[6] M. Atig, et al., "An experimental investigation of heating in induction motor under open phase fault," International Journal of Electrical and Computer Engineering (IJECE), vol. 8, no. 3, pp. 1288-1296, 2018.

[7] X. Chang, et al., "A model of asynchronous machines for stator fault detection and isolation, "IEEE Transactions on Industrial Electronics, vol. 50, no. 3, pp. 578-584, 2003.

[8] R. M. Tallam, et al., "Transient model for induction machines with stator winding turn faults," IEEE Transactions On Industry Applications, vol. 38, no. 3, pp. 632-637, 2002.

[9] M. Hadjami, et al., "Analytical model of cage induction machine dedicated to the study of inner race bearing fault," International Journal of Electrical and Computer Engineering (IJECE), vol. 8, no. 1, pp.458-471, 2018.

[10] A. Ferdjouni, et al., "Observer-based detection of inter-turn short circuit in three phase induction motor stator windings," Mediterranean Journal of Measurement and Control, vol. 2, no. 3, pp. 132-143, 2006.

[11] R. Shahnazi, et al., "Dynamic nonlinear unknown input observer for fault detection of induction motors," 23rd Iranian Conference on Electrical Engineering (ICEE), 2015.

[12] M. Arkan, et al., "Modelling and simulation of induction motors with inter-turn faults for diagnostics," Electric Power Systems Research, vol. 75, pp. 57-66, 2005.

[13] C. S. Kallesoe, et al., "Observer-based estimation of stator-winding faults in delta-connected induction motors: a linear matrix inequality approach," IEEE Transactions on Industry Applications, vol. 43, no. 4, pp. 1022-1031, 2007.

[14] M. S. N. Said, et al., "Detection of broken bars in induction motors using an extended Kalman filter for rotor resistance sensorless estimation," IEEE Transactions on Energy Conversion, vol. 15, pp. 66-70, 2000.

[15] F. Karami, et al., "Detection of broken rotor bars in induction motors using nonlinear Kalman filters," ISA Transactions, vol. 49, pp. 189-195, 2010.

[16] F. Bagheri, et al., "Stator fault detection in induction machines by parameter estimation, using adaptive kalman filter," Control \& Automation, MED '07, Mediterranean Conference on, pp. 1-6, 2007.

[17] K. El Merraoui and A. Ferdjouni, "Detection of inter-turn short circuits in stator windings of im by extended Kalman filters, " 22nd Mediterranean Conference on Control and Automation (MED), pp. 275-280, 2014.

[18] M. E. Zaïm, et al., "Design for torque ripple reduction of a three-phase switched-reluctance machine," IEEE Transactions on Magnetics, vol. 38, no. 2, pp. 1189-1192, 2002.

[19] W. Leonard, Control of Electrical Drives, $2^{\text {nd }}$ ed., Springer-Verlag, Berlin Heidelberg New York, pp. 155-166, 1996.

[20] K. El Merraoui and A. Ferdjouni, "PSO parameters optimization for EKF and AKF for IM rotor speed estimation," Power Electronics and Motion Control Conference and Exposition (PEMC), 16th International, pp. 82-387, 2014.

[21] M. S. Grewal and A. P. Andrews, Kalman Filtering: Theory and Practice Using Matlab, John Wiley \& Sons, pp. 126-133, 2008.

[22] Y. D. Huo, et al., "The parameter optimization of Kalman filter based on multi-objective memetic algorithm," Genetic and Revolutionary computation (GECCO), pp. 613-620, 2014.

[23] K. L. Shi, et al., "Speed estimation of an induction motor drive using an optimized extended Kalman filter," IEEE Trans Ind Electron, vol. 49, pp. 124-133, 2002.

[24] E. Y. Chow and A. S. Willsky, "Analytical redundancy and the design of robust faillure detection systems," IEEE Transactions on automatic Control, vol. AC-29, no. 7, pp. 603-614, 1984.

[25] J. J. Gertler, Fault detection and diagnosis in engineering systems, CRC press, 1998.

[26] P. Frank and X. Ding, "Survey of robust residual generation and evaluation methods in observer-based fault detection systems," Journal of Process Control, vol. 7, no. 6, pp. 403-424, 1997. 


\section{BIOGRAPHIES OF AUTHORS}

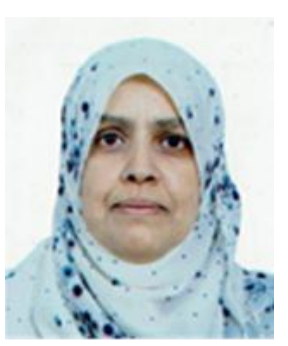

El Merraoui Khadidja is a member of ECME team working on electrical machine control in the SET laboratory at University Saad Dahlab of Blida (USDB), Algeria. She received the Control Engineering degree in 1994 and 'Magiste' degree in 2008 from USDB1. Her main researches include Control and Diagnostic of electric machines and nonlinear systems.

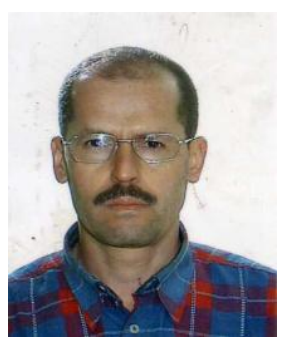

Abdellaziz Ferdjouni is an assistant professor at University Saad Dahleb of Blida 1(USDB1), Algeria. He is a member of ECME team working on electrical machine and diagnostic in the LABSET laboratory in USDB1. He received the "Ingénieur d'état" degree from the "Ecole Nationale Polytechnique" (ENP), Algiers Algeria in 1986, The "Magister" (equiv. MPhil) and "Doctorat d'état" (equiv. PhD) degrees in Control from the USDB1 on 1994 and 2007. Dr Ferdjouni main research interests include Control and Diagnostic of electrical machines, Nonlinear Systems and Chaos

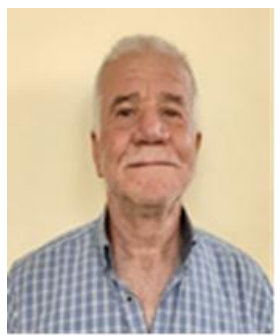

M'hamed Bounekhla is a member of ECME team working on electrical machine control in the SET laboratory at University Saad Dahlab of Blida (USDB); Algeria. He received the electromecanique engineering degree from ENITA Bordj El Bahri; Algeria in 1979, the Master degree in electrical machine from the national polytechnic of Algiers (ENPA), Algeria in 1991 and the $\mathrm{PhD}$. Degree in electrical machine from the national polytechnic school of Algiers (ENPA), Algiers in 2004. He obtain the Professor degree in 2010. His main research interests include nonlinear systems, optimization and identification with application to induction machines. 